\title{
Spatial memory deficits in juvenile rats with pilocarpine induced temporal lobe epilepsy
}

\author{
Orbán-Kis K, Mihály I, Lukács I, Kiss Rita-Judit, Izsák Júlia, Száva Iringó, Metz Júlia, Szilágyi T \\ Department of Physiology, University of Medicine and Pharmacy Tirgu Mures
}

One of the most frequent forms of epilepsy in humans is temporal lobe epilepsy. Characteristic to this form of the disease is the frequent pharmacoresistance and the association with behavioural disorders and cognitive impairment. The objective of our study was to establish the degree of cognitive impairment in a rat model of temporal lobe epilepsy after an initial epileptogenic exposure but before of the onset of the effect of long-duration epilepsy.

Methods. For the experiment we used 11 rats. Status epilepticus was induced by systemic administration of a single dose of pilocarpine. The animals were continuously video-monitored to observe the occurrence of spontaneous recurrent seizures; during weeks 9-10 we performed eight-arm radial maze testing in order to assess the cognitive impairment.

Results. Animals developed spontaneous recurrent seizures after a 14-21 day latency with a daily average seizure density of $0.79 \pm 0.43$ during weeks 9-10. Epileptic rats had significantly more working memory errors per session, more reference memory errors and the number of visited arms was also significantly higher. Accuracy was also lower in the pilocarpine treated group. Interestingly significant differences disappeared after six days of trials.

Conclusions. Our study shows behavioural deficits occurring after 9-10 weeks of epilepsy in the pilocarpine model of epilepsy applied to juvenile rats. In contrast to previous studies, we showed that juvenile rats with short duration of epilepsy are able to learn the behavioural task, therefore a morphopathological and/or behavioural "no-return point" regarding the development of severe cognitive impairment is not reached by status epilepticus alone.

Keywords: epilepsy, hippocampus, cognitive impairment.

Received: 17 October 2014 / Accepted: 25 October 2014

\section{Introduction}

Epilepsy defines a spectrum of disorders that are characterized by the unpredictable occurrence of seizures, widely differing seizure types and causes, and varying impacts on individuals and their families. The disease creates a significant socio-economic burden not only at the individual and family level, but at the societal level, due to the implications regarding health services. Currently there is no cure available, and all therapeutic choices are only symptomatic, with the goal to eliminate or at least to alleviate seizures without interfering with normal function. Data from recent years suggests that by accounting only for seizures, we grossly underestimate the true impact of epilepsy, as the disease is frequently associated with comorbid cognitive and behavioural impairment .

One of the most common forms of epilepsy in humans is temporal lobe epilepsy (TLE), which is also the type (1) most prone to pharmacoresistance and (2) more frequently associated with behavioural disorders and cognitive impairment. TLE causes deficits in declarative memory and in the performance of visuospatial tasks, reduction in psychomotor speed, and executive functions and, on a long term, cognitive deterioration. As such, psychiatric disorders and cognitive impairment in patients with

Correspondence to: Károly Orbán-Kis

E-mail: orbank@gmail.com epilepsy may be considered to reflect the degree of brain dysfunction .

One particularly interesting brain region is the hippocampus which is implicated in memory and learning as well as pathological synchronous oscillations (i.e. epileptic seizures). Therefore the hippocampus may be involved in the same time in temporal lobe epilepsy and the cognitive impairment associated with the disorder.

The cognitive deterioration during chronic epilepsy was described earlier, but there is a lack of data regarding the exact time-course of the development of memory impairment. Therefore, we studied cognitive impairment in a rat model of epilepsy after an initial epileptogenic exposure but before the onset of the effect of long-duration, chronic epilepsy.

\section{Methods}

\section{Animals}

For the experiments we used 11 male Wistar rats (100$250 \mathrm{~g})$ that were housed under standard conditions: constant temperature $\left(21-24^{\circ} \mathrm{C}\right)$ and humidity, 12 hours light/ dark cycle and access to food and water ad libitum. All procedures involving animals were carried out in accordance with EU Directive 2010/63/EU and national and local guidelines and policies (CEC 26/2011). Five 5-6 week old rats were used for status epilepticus induction as well 
as sixe age- and batch matched control animals. Rats were randomly assigned to either control or pilocarpine treated group.

\section{Pilocarpine protocol}

The pilocarpine model of epilepsy was described earlier : rats receiving a single dose of systemic pilocarpine develop status epilepticus (SE); after a period of latency, spontaneous recurrent seizures (SRS) occur that gradually will become more frequent and severe, leading to a form of epilepsy which is analogous to chronic epilepsy in human patients . Five rats received a single injection of methylscopolamine $(1 \mathrm{mg} / \mathrm{kg}$, s.c., necessary to countervail the peripheral effects of the cholinergic agonist) 30 minutes prior to pilocarpine $(350 \mathrm{mg} / \mathrm{kg}$, i.p.) injection. The animals behaviour was observed by experimented researchers for at least 5 hours afterwards. Within the first 10-30 minutes after pilocarpine injection all rats developed seizures with variable severity that evolved into status epilepticus. Seizures were interrupted 2 hours later by administration of Diazepam $(5 \mathrm{mg} / \mathrm{kg}$, i.p.). The duration of SE was selected in accordance with literature data in order to reduce mortality, but in the same time to allow for neural changes characteristic to the model to be established .

\section{Continuous video monitoring}

All rats were continuously video monitored during and after SE for a period of 120-150 days. Night-vision motioncapture camera with I-Catcher software (iCode Systems Ltd, Hampshire, England) was used for video-recording. After the appearance of SRS we established the early seizure pattern and classified seizures according to the modified Racine scale. Video recording were analyzed by several researchers, but, in order to exclude inter-observer differences, a single person reanalyzed all putative SRS found.

\section{Eight-arm radial maze testing}

Rats were tested in the eight-arm radial maze (RAM) according to the procedure described by Olton . First, all animals (control and pilocarpine treated) were partially food deprived until they reached $85 \%$ of their body weight. For testing we used a handmade wooden brown eightarm radial maze with walls but without plastic covering, constructed according to standard specifications. Each of the arms projected from one side of an octagonal center. One small fragment of food pellet was placed at the far end of 6 of the 8 arms before each trial. The same arms remained unbaited throughout the protocol. We did not use reinforcement (i.e. replacement of the acquired pellets). During each trial individual animals were placed in the octagonal center and left in the maze until they had recovered all 6 food baits or until 10 minutes had elapsed, whichever occurred first. Placing all four paws inside an arm was considered entrance. The trial was repeated at least six consecutive days (one test per day), always within the same time interval and same conditions (including visuos- patial clues); times of arm entrances, the identity of each arm entered, the order of arms, the number of baits recovered as well as the time to finish the task was recorded. We analyzed the following parameters: working memory error (WM) - defined as return to a previously visited arm during the same trial, reference memory error (RM) - entry to an unbaited arm after day 2, accuracy of choice (ACC) - number of obtained food pellets from the first 6 visited arms, total number of visited arms (TO) and the number of food pellets obtained during each completed trial (FO). Day one was excluded from analysis (adaptation to the new environment).

\section{Statistical analysis}

Descriptive statistics, nonparametric tests (two-tailed Mann-Whitney) and general linear model were applied for data analysis using GraphPad Prism 5.00 for Windows (GraphPad Software, Inc., San Diego, California, USA). Unless otherwise stated all values are given as Mean \pm SE. A $\mathrm{p}$ value less then 0.05 was considered significant.

\section{Results}

\section{Seizure pattern}

Rats that underwent pilocarpine induction developed SRS after a latency period of 14-21 days. We considered for analysis only rats that developed Racine stage 5 seizures (which is homologous to secondary generalized seizures in humans). The average duration of SRS was $41.9 \pm 15.4$ second $(M e a n \pm S D)$. The number of seizures per day reached $0.79 \pm 0.43$ during weeks 9-10 (all time periods are relative to time of induction with pilocarpine), the period of RAM testing. It must be noted however that the seizure pattern's further evolution was also monitored (i.e. after the RAM testing) to prove the development of chronic epilepsy (Figure 1).

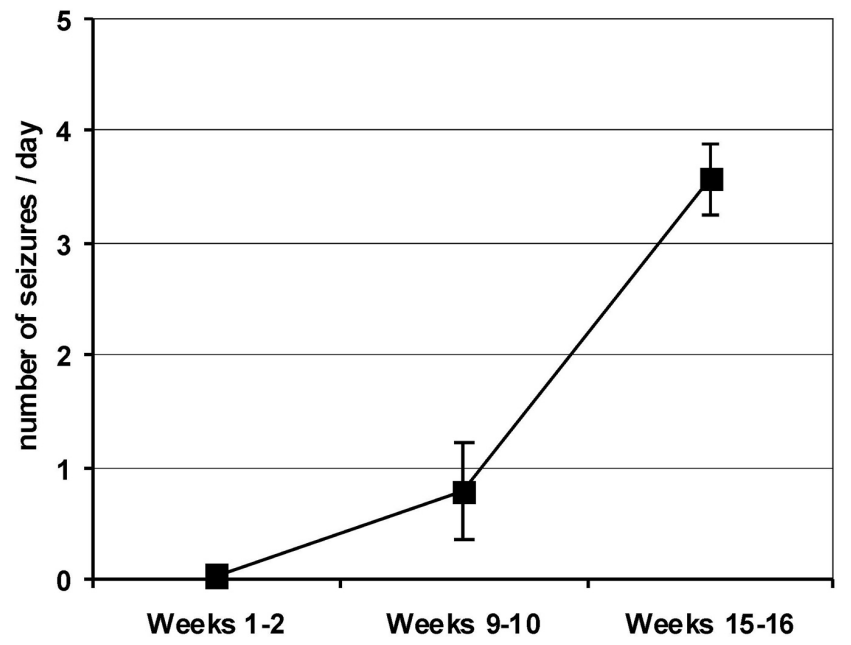

Fig. 1. Seizure pattern progression in rats with induced TLE. 
A

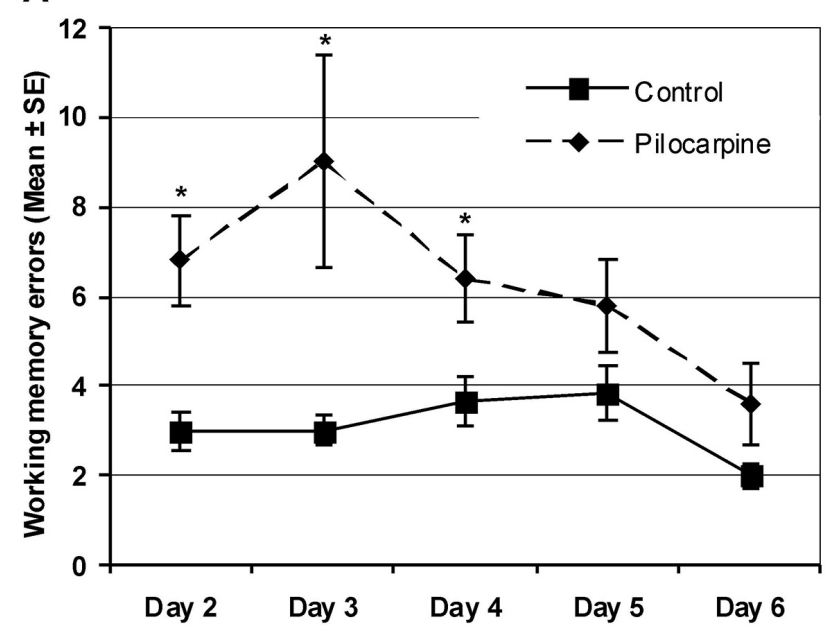

C

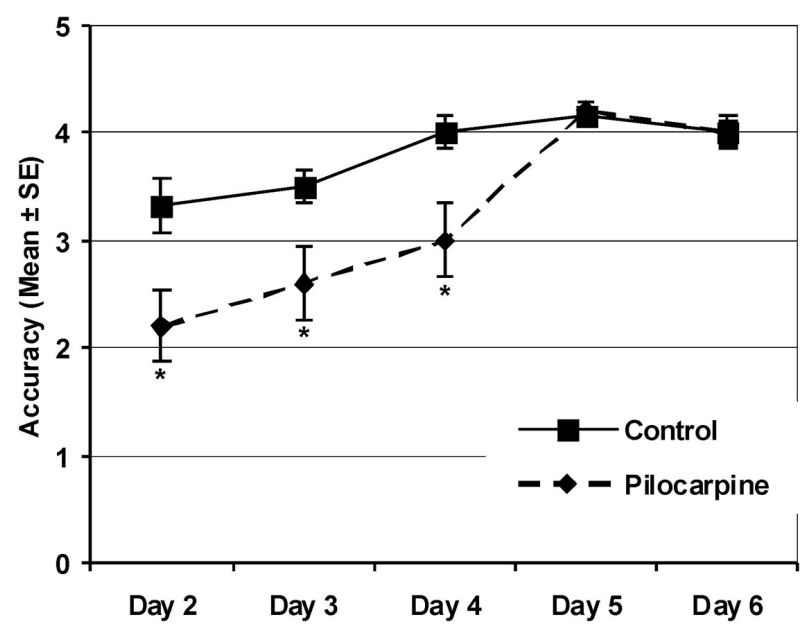

B

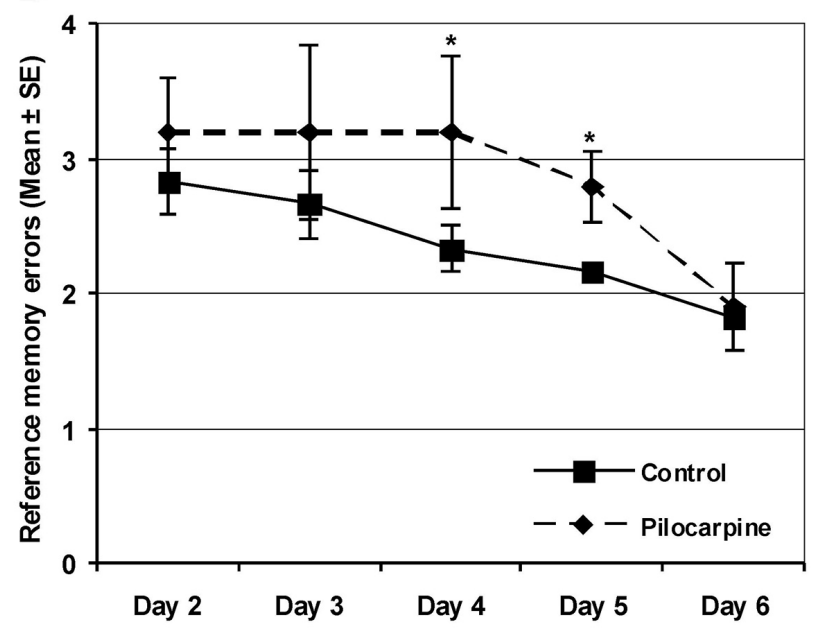

D

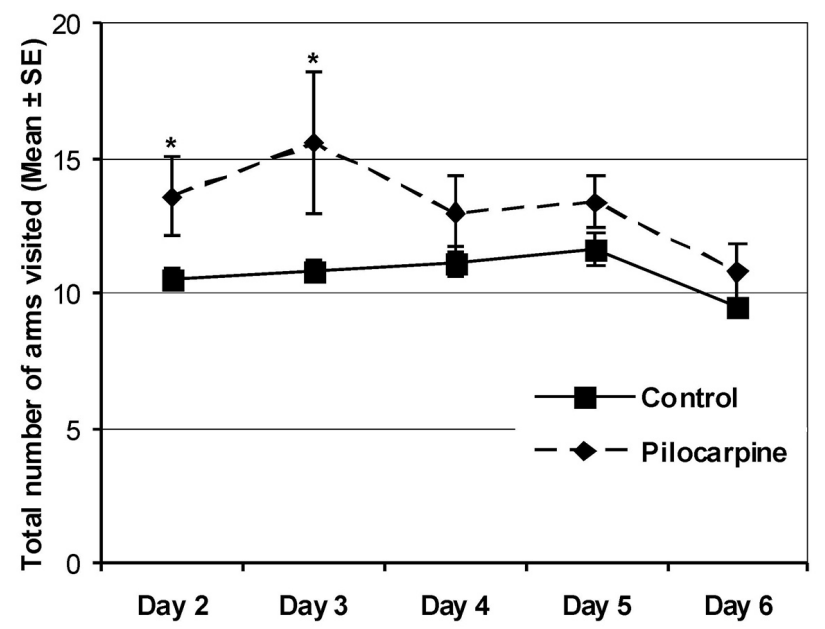

Fig. 2. Behaviour of juvenile rats in the eight-arm radial maze (days marked with * represent statistically significant differences).

Effects of the pilocarpine induced TLE in juvenile rats on the behaviour in the eight-arm radial maze

Rats from the control group learned the task much quicker than rats with TLE as the working and reference memory errors were low starting from day 2 and they further diminished during the 6 day trial period (for summary of results see Figure 2). Furthermore, accuracy was high from the beginning and the rats in this group obtained all food pellets starting from day 2 (Figure 3 ).

Conversely, rats treated with pilocarpine (i.e. rats with TLE) had significantly more working memory errors $(6.4 \pm 0.8$ versus $2.3 \pm 0.3, \mathrm{p}=0.0026)$, more reference memory errors $(2.86 \pm 0.25$ vs. $1.88 \pm 0.22, \mathrm{p}=0.023)$ and the number of visited arms was also significantly higher (13.3 \pm 0.76 vs. $9.7 \pm 0.4, p=0.008)$. Accuracy was lower in the pilocarpine treated group $(3.2 \pm 0.39$ vs. $4.23 \pm 0.17$, $\mathrm{p}=0.04)$ and it took 6 days for rats to be able to collect all food pellets. It must be noted however, that significant differences appeared only during the first days. Most interestingly epileptic rats eventually were able to learn the task, albeit delayed, and on day 6 no significant changes could be found, regardless the analyzed parameter (Figure 2).

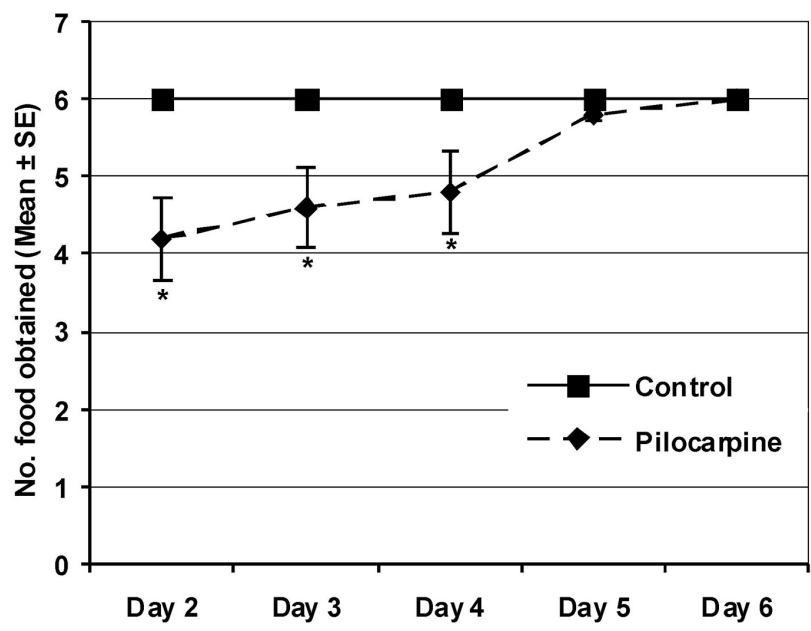

Fig. 3. Food pellets obtained during the 6 day trial period (days marked with * represent statistically significant differences).

\section{Discussion}

Although several papers present the behavioural aspects of TLE in different conditions - immature and adult rats , after a short period or longer period of seizures and with 
different induction age - there is a lack of data regarding the behavioural consequences of SRS in epilepsy induced in juvenile rats (postnatal 4-5 weeks).

All previous studies suggested that spatial learning in epileptic rats is severely impaired. Interestingly, our data suggests that, although learning the task in the eight-arm radial maze is difficult, it is not impossible for juvenile epileptic rats. In our study we observed a gradual improvement of the performance of the epileptic rats along the 6 days of testing. This improvement was characteristic both to the reference and working memory; also the gradual reduction of the number of arms visited showed a learning and goal oriented behaviour, that eventually lead to the collection of all food baits.

In contrast to our study, several groups found that inducing epilepsy in immature rats - postnatal 14-17 days, roughly the equivalent to 6-12 months of human development - causes a permanent reduction in the performance in RAM . This effect however may be caused in conjunction with permanent changes in motor performances . There are morphological, behavioural and psychological evidences that show a link between early cellular alterations and long-term impairment of hippocampal function in adulthood. Furthermore, the development of the dentate gyrus (DG), which plays a significant role in learning , is mainly completed during the first two weeks; as such, it is possible that in previous studies the observed long-term effect on spatial memory is due to the result of seizures in the developing circuitry of the DG .

Other studies looked at the effect of SRS over a long period of time and found that after 5-months duration of epilepsy, rats are unable to learn the task in the RAM, i.e. spatial memory and learning capabilities is adversely affected. The combination of lesions in the hippocampus and in layers II and III of the entorhinal cortex (EC) causes impairment of learning and retention, therefore supporting the poor performance of rats in RAM testing. Furthermore, longer period of SRS significantly worsens the neuronal loss both in the hippocampus and the EC . Therefore, it seems that both SE and SRS contribute to the neuronal loss and the subsequent behavioural impairment in chronic epilepsy.

When shorter periods of SRS were investigated, only in adult animals, severe memory impairment was found with a permanent learning disability . In contrast to this finding, our animals were able to eventually learn the task in RAM, and as the duration of epilepsy was quite short, it seems that the initial trigger (i.e. SE) alone is not enough to disrupt the neuronal network and cause permanent learning disability, at least in juvenile rats. Further studies, morphopathological and chronic performance testing of juvenile rats, are needed to elucidate the onset of "noreturn point" in the development of severe cognitive impairment in epilepsy.

\section{Conclusion}

In conclusion, the present study shows behavioural deficits occurring at 9-10 weeks after induction of epilepsy with pilocarpine in juvenile rats. In contrast to previous studies, we showed that juvenile rats with short-duration epilepsy are able to learn the task in RAM, therefore a morphopathological and/or behavioural "no-return point" regarding the development of permanent learning disability and severe cognitive impairment is not reached by the initial SE alone. Our study also confirms that the pilocarpine model of epilepsy combined with RAM testing is a useful tool to explore behavioural disorders in general and cognitive decline in particular in rats.

\section{Acknowledgements}

This work was supported by the Internal Grant System of the University of Medicine and Pharmacy Tîrgu Mureş, contract number CIGCS 12/2013.

\section{References}

1. Berg AT, Berkovic SF, Brodie MJ et al. Revised terminology and concepts for organization of seizures and epilepsies: report of the ILAE Commission on Classification and Terminology, 2005-2009. Epilepsia. 2010 Apr;51(4):676-685.

2. Pugliatti M, Beghi E, Forsgren $L$ et al. Estimating the cost of epilepsy in Europe: a review with economic modeling. Epilepsia. 2007 Dec;48(12):2224-2233.

3. Glauser T, Ben-Menachem E, Bourgeois B et al. Updated ILAE evidence review of antiepileptic drug efficacy and effectiveness as initial monotherapy for epileptic seizures and syndromes. Epilepsia. 2013 Mar;54(3):551-563.

4. Elger CE, Helmstaedter C, Kurthen M. Chronic epilepsy and cognition. Lancet Neurol. 2004 Nov;3(11):663-672.

5. Helmstaedter C, Aldenkamp AP, Baker GA et al. Disentangling the relationship between epilepsy and its behavioral comorbidities - the need for prospective studies in new-onset epilepsies. Epilepsy Behav. 2014 Feb;31:43-47.

6. Tellez-Zenteno JF, Ronquillo LH, Jette $\mathrm{N}$ et al. Discontinuation of antiepileptic drugs after successful epilepsy surgery. a Canadian survey. Epilepsy Res. 2012 Nov; 102(1-2):23-33.

7. Devinsky O. Therapy for neurobehavioral disorders in epilepsy. Epilepsia. 2004;45 Suppl 2:34-40.

8. Guerreiro CA, Jones-Gotman M, Andermann F et al. Severe Amnesia in Epilepsy: Causes, Anatomopsychological Considerations, and Treatment. Epilepsy Behav. 2001 Jun;2(3):224-246.

9. Abrahams S, Morris RG, Polkey CE et al. Hippocampal involvement in spatial and working memory: a structural MRI analysis of patients with unilateral mesial temporal lobe sclerosis. Brain Cogn. 1999 Oct;41(1):3965.

10. Helmstaedter C, Witt JA. Clinical neuropsychology in epilepsy: theoretical and practical issues. Handb Clin Neurol. 2012;107:437-459.

11. Oyegbile TO, Dow C, Jones $J$ et al. The nature and course of neuropsychological morbidity in chronic temporal lobe epilepsy. Neurology. 2004 May 25;62(10):1736-1742.

12. Lackmayer K, Lehner-Baumgartner E, Pirker $S$ et al. Preoperative depressive symptoms are not predictors of postoperative seizure control in patients with mesial temporal lobe epilepsy and hippocampal sclerosis. Epilepsy Behav. 2013 Jan;26(1):81-86.

13. Clifford DB, Olney JW, Maniotis A et al. The functional anatomy and pathology of lithium-pilocarpine and high-dose pilocarpine seizures. Neuroscience. 1987 Dec;23(3):953-968.

14. Biagini G, Baldelli E, Longo D et al. Proepileptic influence of a focal vascular lesion affecting entorhinal cortex-CA3 connections after status epilepticus. J Neuropathol Exp Neurol. 2008 Jul;67(7):687-701.

15. Curia G, Longo D, Biagini G et al. The pilocarpine model of temporal lobe epilepsy. J Neurosci Methods. 2008 Jul 30;172(2):143-157.

16. Luttjohann A, Fabene PF, van Luijtelaar G. A revised Racine's scale for PTZ-induced seizures in rats. Physiol Behav. 2009 Dec 7;98(5):579-586.

17. Pinel JP, Rovner LI. Electrode placement and kindling-induced 
experimental epilepsy. Exp Neurol. 1978 Jan 15;58(2):335-346.

18. Racine RJ. Modification of seizure activity by electrical stimulation. II. Motor seizure. Electroencephalogr Clin Neurophysiol. 1972 Mar;32(3):281-294.

19. Olton DS, Samuelson RJ. Remembrance of places past: spatial memory in rats. J Exp Psychol Anim Behav Proc. 1976;2:97-116.

20. Wu CL, Huang LT, Liou CW et al. Lithium-pilocarpine-induced status epilepticus in immature rats result in long-term deficits in spatial learning and hippocampal cell loss. Neurosci Lett. 2001 Oct 19;312(2):113-117.

21. Detour J, Schroeder H, Desor D, Nehlig A. A 5-month period of epilepsy impairs spatial memory, decreases anxiety, but spares object recognition in the lithium-pilocarpine model in adult rats. Epilepsia. 2005 Apr;46(4):499508.

22. Persinger MA, Bureau YR, Kostakos $M$ et al. Behaviors of rats with insidious, multifocal brain damage induced by seizures following single peripheral injections of lithium and pilocarpine. Physiol Behav. 1993 May;53(5):849-866.

23. Kinoshameg SE, Persinger MA. Working memory and reference memory in adult rats following limbic seizures induced at 21 or 90 days of age. Psychol Rep. 2002 Dec;91(3 Pt 1):729-730.

24. Pressler R, Auvin S. Comparison of Brain Maturation among Species: An Example in Translational Research Suggesting the Possible Use of Bumetanide in Newborn. Front Neurol. 2013;4:36.

25. Romijn HJ, Hofman MA, Gramsbergen A. At what age is the developing cerebral cortex of the rat comparable to that of the full-term newborn human baby? Early Hum Dev. 1991 Jul;26(1):61-67.

26. Kubova H, Haugvicova R, Suchomelova L, Mares P. Does status epilepticus influence the motor development of immature rats? Epilepsia. 2000;41 Suppl 6:S64-69.
27. Jiang M, Lee CL, Smith KL, Swann JW. Spine loss and other persistent alterations of hippocampal pyramidal cell dendrites in a model of earlyonset epilepsy. J Neurosci. 1998 Oct 15;18(20):8356-8368.

28. Lynch M, Sayin U, Bownds J et al. Long-term consequences of early postnatal seizures on hippocampal learning and plasticity. Eur J Neurosci. 2000 Jul;12(7):2252-2264.

29. Lv J, Xin Y, Zhou W, Qu Z. The epigenetic switches for neural development and psychiatric disorders. J Genet Genomics. 2013 Jul 20;40(7):339-346.

30. Reagh ZM, Watabe J, Ly M et al. Dissociated Signals in Human Dentate Gyrus and CA3 Predict Different Facets of Recognition Memory. J Neurosci. 2014 Oct 1;34(40):13301-13313.

31. Schlessinger AR, Cowan WM, Gottlieb DI. An autoradiographic study of the time of origin and the pattern of granule cell migration in the dentate gyrus of the rat. J Comp Neurol. 1975 Jan 15;159(2):149-175.

32. Myhrer T, Johannesen TS. Learning and retention of a visual discrimination task in rats with various combinations of lesions in the temporalhippocampal region. Brain Res Bull. 1995;36(5):499-503.

33. Dube C, Boyet S, Marescaux C, Nehlig A. Relationship between neuronal loss and interictal glucose metabolism during the chronic phase of the lithium-pilocarpine model of epilepsy in the immature and adult rat. Exp Neurol. 2001 Feb;167(2):227-241. 